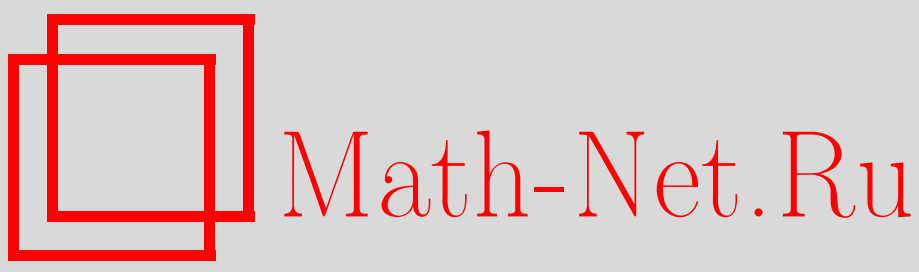

А. А. Арсеньев, Резонансные свойства матрицы рассеяния для одномерного оператора Шрёдингера с ловушечным потенциалом, Матем. сб., 1996, том 187, номер 6, 3-20

DOI: https://doi.org/10.4213/sm133

Использование Общероссийского математического портала Math-Net.Ru подразумевает, что вы прочитали и согласны с пользовательским соглашением

http: //www.mathnet.ru/rus/agreement

Параметры загрузки:

IP : 52.205.19.152

26 апреля 2023 г., 14:39:47 
УДК 517.95

\author{
А.А. Арсеньев
}

\title{
Резонансные свойства матрицы рассеяния для одномерного оператора Шрёдингера с ловушечным потенциалом
}

\footnotetext{
В статье приведено математически строгое описание резонансного рассеяния на одномерном ловушечном потенциале.

Библиографояи: 8 названий.
}

\section{$\S 1$. Введение}

Поясним основные обозначения. Символами $x, y, \ldots$, мы будем обозначать действительные числа: $x, y, \ldots \in \mathbb{R}$, символом $V$ мы будем обозначать функцию $V: \mathbb{R} \rightarrow \mathbb{R}_{+}$со значениями на расширенной полуоси. Тем же символом $V$ мы будем обозначать оператор умножения на функцию $V$. Предположим, что функция $V$ достаточно “хорошая": непрерывна по Гёльдеру на $\mathbb{R}$ и убьвает при $|x| \rightarrow \infty$ быстрее экспоненты.

ОПРЕДЕЛЕниЕ. Решением задачи рассеяния для оператора Шрёдингера

$$
H=-\partial_{x x}^{2}+V
$$

мы называем функции $u^{ \pm}(x, \lambda)$, удовлетворяющие уравнению

$$
H u^{ \pm}=\lambda u^{ \pm}, \quad \lambda>0,
$$

и при $|x| \rightarrow \infty$ имеющие асимптотику

$$
\begin{aligned}
& u^{+}(x, \lambda)=T^{+}(\lambda) \exp (i x \sqrt{\lambda})+o(1), \quad x \rightarrow+\infty, \\
& u^{+}(x, \lambda)=\exp (i x \sqrt{\lambda})+R^{+}(\lambda) \exp (-i x \sqrt{\lambda})+o(1), \quad x \rightarrow-\infty, \\
& u^{-}(x, \lambda)=\exp (-i x \sqrt{\lambda})+R^{-}(\lambda) \exp (i x \sqrt{\lambda})+o(1), \quad x \rightarrow+\infty, \\
& u^{-}(x, \lambda)=T^{-}(\lambda) \exp (-i x \sqrt{\lambda})+o(1), \quad x \rightarrow-\infty
\end{aligned}
$$

Сушествование и единственность решения задачи (1.1), (1.2) хорошо известны, современное учебное изложение этого вопроса есть в [1, с. 116-160].

Коэффициенты $T^{ \pm}(\lambda), R^{ \pm}(\lambda)$ называются коэффициентами прохождения $u$ отражения. Матрицей рассеяния называется матрица, составленная из этих коэффициентов.

Работы выполнена при поддержке Российского фонда фундаменталњных исследований (грант № 93-011-1689). 
В квантовомеханической интерпретации коэффициент $T^{ \pm}(\lambda)$ характеризует вероятность прохождения частицы через барьер $V$, коэффициент $R^{ \pm}(\lambda)$ характеризует вероятность отражения частицы от потенциального барьера. В квантовомеханической интерпретации естественно, что если потенциал $V$ принимает очень большие значения на некотором отрезке $[a, b]$, то модуль коэффициента $T^{ \pm}(\lambda)$ мал, а модуль коэффициента $R^{ \pm}(\lambda)$ примерно равен единице.

$\mathrm{C}$ начала семидесятых годов в связи с математическим моделированием "туннельного диода" и "квантовых проволок" внимание физиков привлек обнаруженный в численных экспериментах и простейших расчетах факт: если потенциал $V$ принимает большие значения на отрезках $[a, b],[c, d]$ и мал в области $[b, c]$, то коэффициенты $R^{ \pm}(\lambda), T^{ \pm}(\lambda)$ при некоторых значениях параметра $\lambda$ имеют резко выраженные резонансы. Модуль коэффициента, $T^{ \pm}(\lambda)$ в резонансе почти равен единице, а модуль коэффициента $R^{ \pm}(\lambda)$ - нулю.

Наша работа посвящена изучению математической модели данного явления. Опишем основную идею применяемых нами методов.

Понятие "очень большое значение потенциала" мы формализуем, вводя потенциал $V_{\infty}$, который принимает бесконечно большие значения на множестве положительной меры, и монотонно возрастаюшую последовательность "хороших" потенциалов $V_{n}, V_{n} \rightarrow V_{\infty}, n \rightarrow \infty$. Задача сводится к исследованию поведения решения задачи (1.1), (1.2) с $V=V_{n}$ при $n \rightarrow \infty$.

Для этого мы должны рассмотреть задачу (1.1), (1.2) при $V=V_{\infty}$. Ясно, что в этой ситуации не работает традиционньй способ сведения дифференциального уравнения к интегральному с помощью функции Грина оператора главной части. Поэтому мы применяем другую стратегию.

По каждому потенциалу $V_{n}$ мы строим самосопряженный оператор

$$
H_{n}=-\partial_{x x}^{2}+V_{n}, \quad H_{0}=-\partial_{x x}^{2}, \quad V_{0}=0,
$$

и рассматриваем последовательность операторов

$$
G_{n}(t)=\exp \left(-t H_{n}\right)
$$

Оператор $G_{n}(t)$ - это интегральный оператор с ядром - функцией Грина оператора теплопроводности (в другой интерпретации - плотность вероятности перехода для процесса броуновского движения с убивающим потенциалом $V_{n}$ ). Из принципа максимума для уравнения теплопроводности сразу же следует, что существует предел

$$
G_{\infty}(t)=\lim G_{n}(t), \quad n \rightarrow \infty
$$

и операторы $G_{\infty}(t)$ образуют полугруппу самосопряженных в $L^{2}(\mathbb{R}, d x)$ операторов. Под оператором

$$
H_{\infty}=-\partial_{x x}^{2}+V_{\infty}
$$

мы по определению понимаем взятый со знаком минус инфинитезимальный оператор полугруппы $G_{\infty}(t)$. Из обшей теории полугрупп следует, что так определенный оператор $H_{\infty}$ самосопряжен. Подчеркнем, что для описания области определения оператора $H_{\infty}$ мы не используем граничные условия.

Прием, состояший в построении самосопряженного расширения предельного оператора как инфинитезимального оператора предельной полугрупп, является 
стандартным в квантовой теории поля, начиная с работ Э. Нельсона шестидесятых годов. Учебное изложение связанных с этим вопросов и ссылки на литературу есть в [2]. Еще до работ Э. Нельсона в теории марковских процессов стандартным приемом было описание инфинитезимального оператора процесса в терминах его переходных вероятностей (чем мы, в сущности, и занимаемся). Но нам нужно не описание области определения инфинитезимального оператора (что обычно составляет предмет исследования), а конструктивный способ описания его специально выбранных собственных функций непрерывного спектра. Принцип инвариантности волновых операторов [3, с. 672] в теории рассеяния позволяет утверждать, что с точностью до естественной замены матрица рассеяния для пары $H_{0}, H_{\infty}$ совпадает с матрицей рассеяния для пары $\exp \left(-t H_{0}\right), \exp \left(-t H_{\infty}\right)$, поэтому нам нужно найти способ описания удовлетворяющих условию излучения собственных функций непрерывного спектра оператора $\exp \left(-t H_{\infty}\right)$. В качестве такого способа предлагается сведение краевой задачи (1.1), (1.2) к интегральному уравнению:

$$
\begin{aligned}
u^{ \pm}(x, \lambda)= & \exp ( \pm i x \sqrt{\lambda})+\varphi^{ \pm}(x, \lambda), \\
\varphi^{ \pm}= & \left(\exp (-(\lambda+i 0) t)-\exp \left(-t H_{0}\right)\right)^{-1} \\
& \times\left(\exp (-t H)-\exp \left(-t H_{0}\right)\right)\left(\exp ( \pm i(\cdot) \sqrt{\lambda})+\varphi^{ \pm}\right) .
\end{aligned}
$$

В уравнение (1.3) потенциал $V$ входит только через $\exp \left(-t\left(H_{0}+V\right)\right)$, поэтому уравнение (1.3) остается "хорошим" для сколь угодно "плохих" неотрицательных потенциалов.

Мы применяем уравнение (1.3) и стандартную технику теории возмущений ограниченных операторов для изучения поведения задачи (1.1), (1.2) при предельном переходе $V_{n} \rightarrow V_{\infty}, n \rightarrow \infty$. При таком подходе принцип максимума для уравнения теплопроводности делает все предельные переходы с идейной точки зрения абсолютно тривиальными. Но поскольку в (1.3) входит произведение операторов, вьписывание всех промежуточных оценок ведет к утомительному выписыванию очень громоздких формул, содержащих оценки интегралов по неравенству Коши-Буняковского. Поэтому мы в ряде мест позволили себе привести лишш формулировку окончательных результатов. Некоторые из приведенных в работе фактов хорошо известны, однако мы формулируем их в виде лемм и теорем для удобства ссылок. Используемые нами методы аналогичны методам, примененным в [4]-[7], а теорема 5.1 аналогична результату, полученному в [6] для абстрактного случая. Новым в данной работе мы считаем два результата: теоремы 5.1, 5.2 и использование уравнения (1.3) в описании решения задачи рассеяния для одномерного оператора Шрёдингера.

Из теоремы 5.1 следует, что для достаточно четко выраженной потенциальной ловушки сушествуют такие точки $\lambda$, в которых коэффициенты $T^{ \pm}(\lambda), R^{ \pm}(\lambda)$ испытывают резкий скачок, а из теоремы 5.2 следует, что для симметричного потенциального барьера коэффициент прохождения в резонансе почти равен единице. Это утверждение и есть основной результат работы.

\section{§2. Вспомогательные построения}

В этом параграфе мы приведем основные определения и некоторые нужные нам утверждения, которые являются либо очевидными следствиями явно выписьваемых формул, либо незначительными переформулировками известных фактов. 
Приведем определение ловушечного потенциала.

ОпреДЕЛЕниЕ. Действительная неотрицательная функция $V_{\infty}: \mathbb{R} \rightarrow \mathbb{R}_{+}$называется ловушечным потенциалом, если она принимает значения на расширенной полупрямой, множество

$$
\omega=\left\{x: V_{\infty}(x)=\infty\right\}
$$

не пусто и есть объединение конечного числа непересекающихся отрезков:

$$
\omega=\bigcup\left[a_{i}, b_{i}\right], \quad 1 \leqslant i \leqslant N, \quad-\infty<a_{i}<b_{i}<\infty,
$$

а функция $\min \left(n, V_{\infty}\right)$ при каждом $n<\infty$ локально удовлетворяет условию Гёльдера и при некотором $r<\infty$ с положительными константами выполнена оценка:

$$
V_{\infty}(x)<C \exp \left(-d x^{2}\right), \quad|x|>r .
$$

В дальнейшем символом $\left\{V_{n}\right\}$ мы обозначим монотонно неубывающую последовательность функций, которые локально удовлетворяют условию Гёльдера и оценке:

$$
\min \left(n, V_{\infty}\right) \leqslant V_{n} \leqslant \min \left(n+1, V_{\infty}\right),
$$

где $V_{\infty}$ - ловушечный потенциал.

При достаточно большом $n$ функцию $V_{n}$ мы также будем называть ловушечным потенциалом.

На множестве $C_{b}^{2}$ всех дважды непрерывно дифференцируемых и ограниченных вместе с производньми функций рассмотрим оператор

$$
H_{n}: u \rightarrow H_{n} u=-\partial_{x x}^{2} u+V_{n} u .
$$

Тем же символом $H_{n}$ при $n<\infty$ мы будем обозначать самосопряженное расширение в $L^{2}(\mathbb{R}, d x)$ оператора, заданного формулой $(2.5)$ на множестве финитных функций.

Пусть

$$
G_{n}(t)=\exp \left(-t H_{n}\right)
$$

где $G_{n}(x, y, t)$ - интегральное ядро оператора $G_{n}(t)$,

$$
\begin{aligned}
G_{0}(x, y, t) & =\frac{1}{\sqrt{4 \pi t}} \exp \left(-\frac{(x-y)^{2}}{4 t}\right), \\
g_{n}(x, y, t) & =G_{0}(x, y, t)-G_{n}(x, y, t),
\end{aligned}
$$

где $g_{n}(x, y, t)$ - интегральное ядро оператора $g_{n}(t)$.

Лемма 2.1. Справедливы оценки

$$
\begin{gathered}
0 \leqslant G_{\infty}(x, y, t) \leqslant G_{n+1}(x, y, t) \leqslant G_{n}(x, y, t) \leqslant G_{0}(x, y, t), \\
0 \leqslant g_{n}(x, y, t) \leqslant g_{n+1}(x, y, t) \leqslant g_{\infty}(x, y, t) \leqslant C \exp \left(-\eta\left(x^{2}+y^{2}\right)\right) .
\end{gathered}
$$

Константы в правой части (2.7) зависят только от $t$ и по определению

$$
\begin{aligned}
g_{\infty}(x, y, t) & =\lim g_{n}(x, y, t), & & n \rightarrow \infty, \\
G_{\infty}(x, y, t) & =\lim G_{n}(x, y, t), & & n \rightarrow \infty .
\end{aligned}
$$


ДокАЗАТЕЛЬСтво. Монотонное убывание и неотрицательность последовательности $G_{n}(x, y, t)$ есть следствие принципа максимума, поэтому в доказательстве нуждается лишш последняя из оценок (2.7).

Две функции Грина $G_{n}^{i}$, отвечающие двум потенциалам $V_{n}^{i}$, удовлетворяют уравнению

$G_{n}^{1}(x, y, t)-G_{n}^{2}(x, y, t)=\int_{0}^{t}\left(\int_{-\infty}^{+\infty} G_{n}^{1}(x, z, t-\tau)\left(V_{n}^{2}(z)-V_{n}^{1}(z)\right) G_{n}^{2}(z, y, \tau) d z\right) d \tau$.

Следовательно, если с не завислщими от $n$ константами выполнено неравенство

$$
0 \leqslant V_{n}^{1}(x)-V_{n}^{2}(x)<C \exp \left(-d x^{2}\right)
$$

то с не зависящими от $n$ константами будет вьполнено неравенство

$$
G_{n}^{1}(x, y, t)-G_{n}^{2}(x, y, t) \leqslant C \exp \left(-\eta\left(x^{2}+y^{2}\right)\right) .
$$

Пусть $\varkappa(x)$ - бесконечно дифференцируемая функция, которая удовлетворяет условиям:

$$
0 \leqslant \varkappa(x) \leqslant 1, \quad \varkappa(x)=1, \quad|x| \leqslant r-1, \quad \varkappa(x)=0, \quad|x| \geqslant r .
$$

Положим

$$
V_{n}^{1}(x)=V_{n}(x) \varkappa(x), \quad V_{n}^{2}(x)=V_{n}(x) .
$$

Тогда из (2.9) следует, что

$$
0 \leqslant G_{\infty}^{1}(x, y, t)-G_{\infty}(x, y, t) \leqslant C \exp \left(-\eta\left(x^{2}+y^{2}\right)\right) .
$$

Обозначая символом $G_{r}^{0}(x, y, t)$ функцию Грина для уравнения теплопроводности с нулевыми граничньми условиями в точках $x= \pm r$, мы получаем

$0 \leqslant G_{0}(x, y, t)-G_{\infty}^{1}(x, y, t) \leqslant G_{0}(x, y, t)-G_{r}^{0}(x, y, t) \leqslant C \exp \left(-\eta\left(x^{2}+y^{2}\right)\right)$.

Складывая два последних неравенства, получаем утверждение леммы.

В дальнейшем числа $a_{i}, b_{i}$ определены формулой (2.2).

Положим

$$
b_{0}=-\infty, \quad a_{N+1}=\infty .
$$

Пространства $L^{2}\left(\left(b_{i}, a_{i+1}\right), d x\right)$ мы будем рассматривать как подпространства $L^{2}(\mathbb{R}, d x)$, состоящие из равных нулю вне $\left(b_{i}, a_{i+1}\right)$ функций.

Теорема 2.1. Интегральнье операторь $G_{\infty}(t)$ с ядром $G_{\infty}(x, y, t)$ в $L^{2}(\mathbb{R}, d x)$ образуют полугруппу самосопряженных операторов. Каждое из пространств $L^{2}\left(\left(b_{i}, a_{i+1}\right), d x\right)$ приводит полугруппу $G_{\infty}(t)$, и в каждом из них она принадлежит классу $C_{0}$. Инфинитезимальный оператор полугруппь $G_{\infty}(t)$ самосопряжен в $L^{2}(C \omega, d x)$ и в каждом из пространств $L^{2}\left(\left(b_{i}, a_{i+1}\right), d x\right)$. На финитных в $C \omega$ функциях он вычисляется по формуле

$$
\left.\partial_{t} G_{\infty}(t) \varphi\right|_{t=0}=\partial_{x x}^{2} \varphi-V_{\infty} \varphi
$$


ДоКАЗАТЕЛЬСТВо. Из оценки $(2.6)$ и симметрии функции $Г$ рина $G_{n}(x, y, t)$ следует, что интегральный оператор $G_{\infty}(t)$ самосопряжен в каждом из пространств $L^{2}\left(\left(b_{i}, a_{i+1}\right), d x\right)$. Функция $G_{n}(x, y, t)$ может быть вычислена по формуле

$G_{n}(x, y, t)=G_{0}(x, y, t) E\left(\exp \left(-t \int_{0}^{1} V_{n}(2 \sqrt{t}(x(\tau)-\tau x(1))+x+(y-x) \tau) d \tau\right)\right)$,

где символом $E(\ldots)$ мы обозначили интеграл по мере Винера от функционала $x(\tau) \rightarrow F(x(\tau))$ броуновской траектории $\tau \rightarrow x(\tau), 0 \leqslant \tau \leqslant 1$.

Так как с вероятностью единица броуновская траектория непрерывна, функция $G_{\infty}(x, y, t)$ равна нулю, если точки $x, y$ принадлежат разным компонентам связности множества $C \omega$, или если одна из них принадлежит внутренности $\omega$. Отсюда следует, что оператор $G_{\infty}(t)$ приводит каждое из пространств $L^{2}\left(\left(b_{i}, a_{i+1}\right), d x\right)$. Полугрупповое тождество для оператора $G_{\infty}(t)$ следует из полугруппового тождества для $G_{n}(t)$, формула (2.10) для инфинитезимального оператора следует из того факта, что в дополнении к любой окрестности множества $\omega$ функция $G_{\infty}(x, y, t)$ есть решение уравнения теплопроводности с потенциалом $V_{\infty}$.

Положим по определению

$$
H_{\infty}=-\left.\partial_{t} G_{\infty}(t)\right|_{t=0} .
$$

Так как любая финитная в $C \omega$ функция принадлежит области определения оператора $H_{\infty}$, полугруппа $C_{\infty}(t)$ есть полугруппа самосопряженных операторов класса $C_{0}$ в $L^{2}(C \omega, d x)$ и в $L^{2}\left(\left(b_{i}, a_{i+1}\right), d x\right)$, а оператор $H_{\infty}$ самосопряжен в $L^{2}(C \omega, d x)$ и в каждом из пространств $L^{2}\left(\left(b_{i}, a_{i+1}\right), d x\right)$. Теорема доказана.

Теорема 2.2. При любом $а>0$ и $n<\infty$ интегральные операторы $G_{n}(t)$ образуют полугруппу класса $C_{0}$ в $L^{1}(\mathbb{R}, \exp (-a|x|) d x)$. Интегральные операторы $G_{\infty}(t)$ образуют полугруппу класса $C_{0}$ в $L^{1}(C \omega, \exp (-a|x|) d x)$.

ДокАЗАТЕльство. Достаточно заметить, что оператор $G_{0}(t)$ ограничен в $L^{1}(\mathbb{R}, \exp (-a|x|) d x)$ при любом $a>0$.

В дальнейшем мы будем считать, что параметр $a$ выбран достаточно большим.

В следуюших ниже рассуждениях параметр $n$ может принимать любые значения, включая $n=\infty$.

Tеорема 2.3. Если функиия и из $L^{\infty}(\mathbb{R}, d x)$ при каком-нибудь $t>0$ есть решение уравнения

$$
\exp (-\lambda t) u=G_{n}(t) u, \quad|\operatorname{Im} \lambda| \leqslant \frac{\pi}{t},
$$

то функиия и удовлетворяет этому уравнению при всех $t>0$ и поэтому удовлетворяет уравнению

$$
H_{n} u=\lambda u,
$$

где при $n<\infty$ под $H_{n}$ понимается инфинитезимальный оператор полугруппь $G_{n}(t)$ в $L^{1}(\mathbb{R}, \exp (-a|x|) d x)$, а при $n=\infty$ понимается инфинитезимальный оператор полугруппы $G_{\infty}(t)$ в $L^{1}(C \omega, \exp (-a|x|) d x)$.

Это утверждение есть переформулировка для рассматриваемого нами случая известного факта теории полугрупп класса $A$ [8, теорема 16.7.2]. 
Положим

$$
\begin{gathered}
\left(\exp (-\lambda t)-G_{0}(t)\right)^{-1}=\exp (\lambda t)(E+K(\lambda)) \\
\lambda \notin[0, \infty) \bmod (2 \pi i t) .
\end{gathered}
$$

При преобразовании Фурье оператор $G_{0}(t)$ переходит в оператор умножения на $\exp \left(-k^{2} t\right)$.

Перенося в формуле обрашения контур интегрирования на гиперболу $\operatorname{Im} k^{2}=$ $-\sigma<0$, мы получаем, что оператор $K(\lambda)$ в $(2.13)$ можно представить в виде

$$
K(\lambda)=-\frac{1}{t} R\left(\lambda, H_{0}\right)+A(\lambda)
$$

г де $R\left(\lambda, H_{0}\right)$ интегральный оператор с ядром

$$
R\left(\lambda, H_{0}\right)(x, y)=-\frac{i}{2 \sqrt{\lambda}} \exp (i|x-y| \sqrt{\lambda}), \quad 0<\arg (\sqrt{\lambda})<\pi
$$

И

$$
A(\lambda)=-\frac{1}{2 \pi i} \int \exp (-\mu t)(\exp (-\lambda t)-\exp (-\mu t))^{-1} R\left(\mu, H_{0}\right) d \mu
$$

Интеграл в (2.15) берется по параболе

$$
\operatorname{Re} \mu=\frac{1}{4} \sigma^{-2}(\operatorname{Im} \mu)^{2}-\sigma^{2}
$$

и $\sigma$ выбрано настолько малым, что круг $\left|\lambda-\lambda_{0}-2 \pi i\right|<\delta$ лежит выше параболы, а круг $\left|\lambda-\lambda_{0}\right|<\delta$ лежит между ветвей параболы.

Из (2.14) и (2.15) следует

Теорема 2.4. Каково би ни било $\lambda_{0}>0$, существует такое $\delta>0$, что в круге $\left|\lambda-\lambda_{0}\right|<\delta$ оператор $K(\lambda)$ аналитичен по $\lambda$ как оператор из $L^{1}(\mathbb{R}, \exp (a|x|) d x)$ в $L^{1}(\mathbb{R}, \exp (-a|x|) d x)$, и в круге $\left|\lambda-\lambda_{0}\right|<\delta$ справедливо равенство

$$
\left(\exp (-\lambda t)-G_{0}(t)\right) \exp (\lambda t)(E+K(\lambda)) \psi=\psi, \quad \psi \in L^{1}(\mathbb{R}, \exp (a|x|) d x)
$$

ДокАЗАТЕльство. В нем нуждается лишш формула (2.16) при $\operatorname{Im} \lambda \leqslant 0$, а она получается аналитическим продолжением из области $\operatorname{Im} \lambda>0$ с учетом того факта, что оператор $\left(\exp (-\lambda t)-G_{0}(t)\right)$ аналитичен по $\lambda$ в $L^{1}(\mathbb{R}, \exp (-a|x|) d x)$.

Предположим, что $g$ - интегральный оператор, ядро которого удовлетворяет оценке (2.7), $u \in L^{1}(\mathbb{R}, \exp (-a|x|) d x)$.

Теорема 2.5. Справедливь следующие утверждения:

1) функиия

$$
I(x, \lambda)=-\exp (\lambda t)(E+K(\lambda)) g u(x)
$$

в области $\{\lambda: \operatorname{Re} \lambda>0,0<\operatorname{Im} \lambda \leqslant \pi / t\}$ принадлежсит $L^{\infty}(\mathbb{R}, d x)$ и при $|x| \rightarrow \infty$ убъвает бъстрее любой степени $1 /|x|$; 
2) в области $\{\lambda: \operatorname{Re} \lambda>0,-\pi / t \leqslant \lambda \leqslant 0\}$ функция $I(x, \lambda)$ удовлетворяет оценке

$$
|I(x, \lambda)| \leqslant C \exp \left(\frac{\sqrt{\pi}}{t}|x|\right)
$$

3) при $\lambda>0$ функция $I(x, \lambda)$ ограничена по $x$ и при $|x| \rightarrow \infty$ имеет асимптотику

$$
\begin{aligned}
I(x, \lambda)=-\frac{1}{2 t \sqrt{\lambda}} & \exp (\lambda t+i|x| \sqrt{\lambda}) \\
& \times \int_{-\infty}^{+\infty} \exp (\mp i y \sqrt{\lambda}) g u(y) d y+o(1), \quad x \rightarrow \pm \infty
\end{aligned}
$$

где о(1) убъвает бъстрее любой степени $1 /|x|$.

ДокАЗАТЕЛЬСтво. Утверждение теоремы есть очевидное следствие формул $(2.14)$ и $(2.15)$.

Положим

$$
\begin{aligned}
F_{n}(\lambda) & =\left(\exp (-\lambda t)-G_{0}(t)\right)^{-1}\left(G_{n}(t)-G_{0}(t)\right) \\
& =-\exp (\lambda t)(E+K(\lambda)) g_{n}(t), \quad 0<\operatorname{Im} \lambda \leqslant \frac{\pi}{t} .
\end{aligned}
$$

ТЕОРема 2.6. В ядерной топологии пространства

$$
L^{2}(\mathbb{R}, \exp (-a|x|) d x) \rightarrow L^{2}(\mathbb{R}, \exp (-a|x|) d x)
$$

оператор $F_{n}(\lambda)$ аналитичен по $\lambda$ для $\operatorname{Re} \lambda>0,|\operatorname{Im} \lambda| \leqslant \pi / t$. При $n \rightarrow \infty$ оператор $F_{n}(\lambda)$ сходится в ядерной топологии к оператору $F_{\infty}(\lambda)$ равномерно по $\lambda$ на компактах.

ДоКАЗАТЕЛЬСтво. Пусть $B$ - оператор умножения на $\exp (-b|x|), b>0$. Справедливо равенство

$$
\begin{aligned}
g_{n}(t) & =G_{0}(t)-G_{n}(t) \\
& =\left(g_{n}\left(\frac{t}{2}\right) B^{-1}\right)\left(B G_{0}\left(\frac{t}{2}\right)\right)+\left(G_{n}\left(\frac{t}{2}\right) B\right)\left(B^{-1} g_{n}\left(\frac{t}{2}\right)\right) .
\end{aligned}
$$

Если параметр $b$ выбран достаточно большим, то каждый из операторов $(\ldots)$ в (2.18) принадлежит классу Гильберта-Шмидта в $L^{2}(\mathbb{R}, \exp (-a|x|) d x)$.

Операторы

$$
K(\lambda) g_{n}\left(\frac{t}{2}\right) B^{-1}, \quad K(\lambda) G_{n}\left(\frac{t}{2}\right) B
$$

принадлежат классу Гильберта-Шмидта, аналитичны по $\lambda$ в области $\operatorname{Im} \lambda>0$ и имеют аналитическое продолжение в область $\operatorname{Im} \lambda \leqslant 0$. Сходимость операторов $F_{n}(\lambda)$ при $n \rightarrow \infty$ очевидна. 


\section{§ 3. Исследование основного интегрального уравнения}

В $L^{2}(\mathbb{R}, \exp (-a|x|) d x)$ рассмотрим уравнение

$$
\mu \psi=F_{n}(\lambda) \psi, \quad \lambda \in(0, \infty) .
$$

Введем билинейную форму

$$
\langle f \mid \varphi\rangle=\int_{-\infty}^{+\infty} f(x) \varphi(x) d x .
$$

Теорема 3.1. Решение уравнения (3.1) удовлетворяет равенству

$$
\begin{aligned}
\left|\left\langle g_{n} \psi \mid \exp (i(\cdot) \sqrt{\lambda})\right\rangle\right|^{2}+\mid\left\langle g_{n} \psi\right| & \exp (-i(\cdot) \sqrt{\lambda})\rangle\left.\right|^{2} \\
& =-4 t \sqrt{\lambda} \exp (-\lambda t)(\operatorname{Im} \mu)\left\langle g_{n} \psi \mid \psi\right\rangle .
\end{aligned}
$$

ДокАЗАТЕЛЬСТво. Из (3.1) следует, что

$$
\begin{aligned}
\mu\left\langle g_{n} \bar{\psi} \mid \psi\right\rangle-\bar{\mu}\left\langle g_{n} \psi \mid \bar{\psi}\right\rangle= & 2 i(\operatorname{Im} \mu)\left\langle g_{n} \bar{\psi} \mid \psi\right\rangle \\
= & -i \frac{\exp (\lambda t)}{2 t \sqrt{\lambda}}\left(\left|\left\langle g_{n} \psi \mid \exp (i(\cdot) \sqrt{\lambda})\right\rangle\right|^{2}\right. \\
& \left.+\left|\left\langle g_{n} \psi \mid \exp (-i(\cdot) \sqrt{\lambda})\right\rangle\right|^{2}\right) .
\end{aligned}
$$

Последнее равенство есть следствие формулы Сохоцкого, примененной к резольвенте оператора $G_{0}(t)$.

СлЕДСТвИЕ 3.1. При $\lambda \in(0, \infty)$ решение уравнения

$$
\psi=F_{n}(\lambda) \psi, \quad \psi \in L^{2}(\mathbb{R}, \exp (-a|x|) d x)
$$

удовлетворяет равенству

$$
\left|\left\langle g_{n} \psi \mid \exp (i(\cdot) \sqrt{\lambda})\right\rangle\right|^{2}+\left|\left\langle g_{n} \psi \mid \exp (-i(\cdot) \sqrt{\lambda})\right\rangle\right|^{2}=0 .
$$

Теорема 3.2. При $0 \leqslant \operatorname{Im} \lambda \leqslant \pi / t, \lambda \neq 0$, решение уравнения (3.4) удовлетворяет уравнению (2.12).

ДоказАТЕЛЬСтво. Если функция $\psi \in L^{2}(\mathbb{R}, \exp (-a|x|) d x)$, то функция $g_{n} \psi \in L^{1}(\mathbb{R}, \exp (a|x|) d x)$, и мы можем умножить обе части (3.3) на $\exp (\lambda t)(E+K(\lambda))$, а потом воспользоваться теоремой 2.4. Получим, что решение уравнения (3.4) удовлетворяет уравнению (2.11) и поэтому уравнению (2.12).

Положим

$$
\omega 2=\bigcup\left(b_{i}, a_{i+1}\right), \quad 1 \leqslant i<N, \quad \omega 1=\left(-\infty, a_{1}\right) \cup\left(b_{N}, \infty\right) .
$$

Заметим, что в $L^{2}(\omega 2, d x)$ оператор $G_{\infty}(t)$ вполне непрерывен, поэтому спектр сужения оператора $H_{\infty}$ дискретен. Мы обозначим его через $\left\{\lambda_{j}\right\}$. 
Теорема 3.3. При $0<\operatorname{Im} \lambda \leqslant \pi / t$ уравнение (3.4) имеет только тривиальное решение. При $\lambda \in(0, \infty)$ уравнение (3.4) имеет нетривиальное решение лишь при $n=\infty, \lambda \in\left\{\lambda_{j}\right\}$, и это решение есть собственная функция дис-

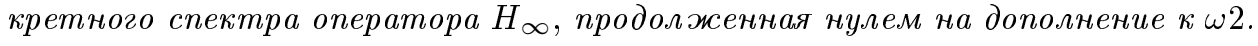

ДокАЗАТЕЛЬСТво. Если $0<\operatorname{Im} \lambda \leqslant \pi / t$, то решение уравнения (3.4) принадлежит $L^{2}(\mathbb{R}, d x)$ и удовлетворяет равенству $(2.11)$, поэтому оно равно нулю. Если $\lambda \in(0, \infty)$, то решение уравнения (3.4) удовлетворяет уравнению (2.12) и в силу теоремы 2.5 и следствия 3.1 при $|x| \rightarrow \infty$ убывает быстрее любой степени $1 /|x|$, поэтому оно равно нулю на $\omega 1$ при $n=\infty$ и равно нулю всюду, если $n<\infty$. Если $n=\infty$ и $\lambda \notin\left\{\lambda_{j}\right\}$, то решение уравнения (3.4) равно нулю, так как оно должно удовлетворять уравнению (2.11), а если $n=\infty, \lambda \in\left\{\lambda_{j}\right\}$, то оно либо нуль, либо совпадает с собственной функцией оператора $H_{\infty}$, продолженной нулем на дополнение к $\omega 2$. Теперь предположим, что $\psi\left(x, \lambda_{j}\right)$ - собственная функция оператора $H_{\infty}$, продолженная нулем на дополнение к $\omega 2$. Тогда она при всех $t>0$ и $n=\infty$ удовлетворяет уравнению (2.11), а поэтому и уравнению

$$
\left(\exp \left(-\lambda_{j} t\right)-G_{0}(t)\right) \psi\left(\cdot, \lambda_{j}\right)=-g_{\infty}(t) \psi\left(\cdot, \lambda_{j}\right)
$$

Применив теорему 2.4, мы получим, что $\psi\left(x, \lambda_{j}\right)$ удовлетворяет уравнению (3.4).

Из теорем 3.3 и 2.6 вытекает

СлЕДСтвиЕ 3.2. При $n<\infty$ операторная функиия

$$
\lambda \rightarrow\left(E-F_{n}(\lambda)\right)^{-1}
$$

аналитична по $\lambda$ в окрестности каждой точки $\lambda \in(0, \infty)$ как оператор в $L^{2}(\mathbb{R}, \exp (-a|x|) d x)$. При $n=\infty$ операторная функиия (3.6) аналитична по $\lambda$ в окрестности каждой точки $\lambda \in(0, \infty) \backslash\left\{\lambda_{j}\right\}$, и каждая точка $\lambda_{j}$ есть полюс операторной функции (3.6). Операторная функция

$$
\mu \rightarrow\left(\mu E-F_{\infty}\left(\lambda_{j}\right)\right)^{-1}
$$

в точке $\mu=1$ имеет полюс.

Начиная с этого места, мы предположим, что $\lambda_{j}-$ фиксированное простое собственное значение оператора $H_{\infty}$ в $L^{2}(\omega 2, d x)$. Тогда в $L^{2}(\mathbb{R}, \exp (-a|x|) d x)$ размерность пространства решений уравнения

$$
\psi=F_{\infty}\left(\lambda_{j}\right) \psi
$$

равна единице, нормированное нетривиальное решение уравнения (3.7) совпадает с собственной функцией оператора $H_{\infty}$ на $\omega 2$ и равно нулю на $\omega 1$. Обозначим это решение через $\psi\left(x, \lambda_{j}\right)$. 
ТЕОРема 3.4. Точка $\mu=1$ есть алгебраически простое собственное значение оператора $F_{\infty}\left(\lambda_{j}\right)$, m.e.

а) она есть полюс первого порядка для резольвенты $R\left(\mu, F_{\infty}\left(\lambda_{j}\right)\right)$;

б) размерность области значений проектора

$$
P\left(\lambda_{j}\right)=\frac{1}{2 \pi i} \int_{|1-\mu|=\delta} R\left(\mu, F_{\infty}\left(\lambda_{j}\right)\right) d \mu
$$

при $0<\delta<\delta_{0}$ и $\delta_{0}$ достаточно малом равна единице.

Заметим, что в рассматриваемом нами случае геометрически простого собственного значения, утверждения а) и б) эквивалентны [3, с. 60, задачи 5.12 и 5.13]. Доказательство теоремы 3.4 приведено в [4, теорема 8].

Рассмотрим проектор

$$
P_{j, n}(\lambda)=\frac{1}{2 \pi i} \int_{|1-\mu|=\delta} R\left(\mu, F_{n}\left(\lambda_{j}\right)\right) d \mu .
$$

Так как при $n \rightarrow \infty$ оператор $F_{n}(\lambda) \rightarrow F_{\infty}(\lambda)$ равномерно по $\lambda$ на компактах, то очевидна

Лемма 3.1. Существуют такие $\varepsilon>0$ u $n_{0}<\infty$, ито при $n>n_{0} u$ $\left|\lambda-\lambda_{j}\right|<\varepsilon$ размерность области значений проектора (3.9) равна единице.

Из леммы 3.1 вытекает

СЛЕДСТвИЕ 3.3. При $n>n_{0} u\left|\lambda-\lambda_{j}\right|<\varepsilon$ у оператора $F_{n}(\lambda)$ в круге $|1-\mu|<\delta_{0}$ есть точно одно собственное значение $\mu_{n}(\lambda)$. Алгебрачческая кратность собственного значения $\mu_{n}(\lambda)$ равна единице, поэтому функция $\mu \rightarrow R\left(\mu, F_{n}(\lambda)\right)$ в точке $\mu_{n}(\lambda)$ имеет полюс первого порядка. Функция $\mu_{n}(\lambda)$ аналитична по $\lambda$ в круге $\left|\lambda-\lambda_{j}\right|<\varepsilon$.

Последнее утверждение есть следствие формулы

$$
\mu_{n}(\lambda)=\operatorname{tr}\left(P_{n, j}(\lambda) F_{n}(\lambda)\right)
$$

Из этой же формулы следует, что

$$
\mu_{n}(\lambda) \rightarrow \mu_{\infty}(\lambda), \quad n \rightarrow \infty,
$$

равномерно по $\lambda$ в круге $\left|\lambda-\lambda_{j}\right|<\varepsilon$.

Пусть $\psi(\cdot \mid \lambda, n)$ - собственная функция оператора $F_{n}(\lambda)$, которая отвечает собственному значению $\mu_{n}(\lambda)$ :

$$
\mu_{n}(\lambda) \psi(\cdot \mid \lambda, n)=F_{n}(\lambda) \psi(\cdot \mid \lambda, n) .
$$

Заметим, что ее можно выбрать так, что она будет аналитична по $\lambda$, непрерьвна по $n$ и будет удовлетворять соотношению: $\psi\left(x \mid \lambda_{j}, \infty\right)=\psi\left(x, \lambda_{j}\right)$, где $\psi\left(x, \lambda_{j}\right)-$ собственная функция оператора $H_{\infty}$, продолженная нулем на дополнение к $\omega 2$. 
Лемма 3.2. Справедлива формула

$$
\left.\frac{\partial \mu_{\infty}(\lambda)}{\partial \lambda}\right|_{\lambda=\lambda_{j}}=\frac{t \exp \left(-\lambda_{j} t\right)}{\left\langle\psi\left(\cdot, \lambda_{j}\right) \mid\left(\exp \left(-\lambda_{j} t\right)-G_{0}(t)\right) \psi\left(\cdot, \lambda_{j}\right)\right\rangle},
$$

где $\psi\left(x, \lambda_{j}\right)$ нормирована на единицу относительно формы (3.1).

Доказательство этой леммы приведено в $[4$, лемма 4$]$.

Из леммы 3.2 следует, что

$$
\left.\frac{\partial \mu_{\infty}(\lambda)}{\partial \lambda}\right|_{\lambda=\lambda_{j}} \neq 0
$$

поэтому справедлива

Лемма 3.3. Можно так выбрать $\varepsilon>0$ u $n_{0}<\infty$, ито при $n>n_{0} u$ $\left|\lambda-\lambda_{j}\right|<\varepsilon$ уравнение

$$
1-\mu_{n}(\lambda)=0
$$

будет иметь точно один корень, который в дальнейшем мы будем обозначать через $\lambda_{j, n}$. Этот корень таков, что

$$
\begin{gathered}
\operatorname{Im} \lambda_{j, n}<0, \quad n<\infty ; \quad \lambda_{j, n} \rightarrow \lambda_{j}, \quad n \rightarrow \infty \\
\left.\frac{\partial \mu_{n}(\lambda)}{\partial \lambda}\right|_{\lambda=\lambda_{j, n}} \neq 0 .
\end{gathered}
$$

ДокАЗАТЕльство. Все утверждения леммы очевидны, за исключением первого неравенства, а оно следует из того факта, что при $0<\operatorname{Im} \lambda \leqslant \pi / t$ и $n<\infty$ уравнение (3.1) нетривиальных решений не имеет.

ЛЕмма 3.4. Проектор (3.9) может быть вычислен по формуле

$$
P_{j, n} \varphi=C_{n}^{-1}(\lambda) \psi(\cdot \mid \lambda, n)\left\langle\varphi \mid\left(\exp (-\lambda t)-G_{0}(t)\right) \psi(\cdot \mid \lambda, n)\right\rangle,
$$

zдe

$$
C_{n}(\lambda)=\left\langle\psi(\cdot \mid \lambda, n) \mid\left(\exp (-\lambda t)-G_{0}(t)\right) \psi(\cdot \mid \lambda, n)\right\rangle .
$$

ДокАЗАТЕЛЬСТво. Положим

$$
\beta(\cdot \mid \lambda, n)=\left(\exp (-\lambda t)-G_{0}(t)\right) \psi(\cdot \mid \lambda, n) .
$$

Из (3.10) следует, что справедлива оценка

$$
|\beta(x \mid \lambda, n)|<C \exp \left(-\eta x^{2}\right) .
$$

Из этой оценки и теоремы 2.4 следует равенство

$$
\mu_{n}(\lambda) \beta=-g_{n}\left(\exp (-\lambda t)-G_{0}(t)\right)^{-1} \beta,
$$

поэтому $\beta$ есть собственная функция оператора, сопряженного к $F_{n}(\lambda)$ относительно формы (3.2), а отсюда и вытекает формула для проектора (3.9).

Следствием наших рассуждений является 
ТЕОРема 3.5. Если $\psi\left(x, \lambda_{j}\right)$ - простая собственная функиия оператора $H_{\infty}$, то существуют такие $\varepsilon>0$ u $n_{0}<\infty$, ито при $n>n_{0} u\left|\lambda-\lambda_{j}\right|<\varepsilon$ в $L^{2}(\mathbb{R}, \exp (-a|x|) d x)$ справедливо равенство

$$
\left(E-F_{n}(\lambda)\right)^{-1}=\left(1-\mu_{n}(\lambda)\right)^{-1} P_{j, n}(\lambda)+S_{n}(\lambda),
$$

әде проектор $P_{j, n}(\lambda)$ описан леммой 3.4. Oператоры $P_{j, n}(\lambda)$ u $S_{n}(\lambda)$ аналитичны по $\lambda$ в круге $\left|\lambda-\lambda_{j}\right|<\varepsilon$ и равномерно по $\lambda$ :

$$
S_{n}(\lambda) \rightarrow S_{\infty}(\lambda), \quad P_{j, n}(\lambda) \rightarrow P_{j, \infty}(\lambda), \quad \mu_{n}(\lambda) \rightarrow \mu_{\infty}(\lambda) .
$$

\section{§4. Задача рассеяния}

Определение решения задачи рассеяния было дано в 1 .

ТЕОРЕМА 4.1. Пусть при некотором $t>0$ функиия $\varphi_{n}^{+}(x, \lambda)$ есть принадлежсачее $L^{2}(\mathbb{R}, \exp (-a|x|) d x)$ решение уравнения:

$$
\varphi_{n}^{+}(\cdot, \lambda)=F_{n}(\lambda)\left(\exp (i(\cdot) \sqrt{\lambda})+\varphi_{n}^{+}(\cdot, \lambda)\right), \quad 0<\lambda<\infty .
$$

Тогда функиия

$$
u_{n}^{+}(x, \lambda)=\exp (i x \sqrt{\lambda})+\varphi_{n}^{+}(x, \lambda)
$$

есть решение задачи рассеяния.

ДокАзАтЕльство. Умножим обе части (4.1) на $\left(\exp (-\lambda t)-G_{0}(t)\right)$. В силу теоремы 2.4 получим, что определенная равенством (4.2) функция $u_{n}^{+}(x, \lambda)$ удовлетворяет равенству (2.11) и поэтому в силу теоремы 2.3 удовлетворяет уравнению (1.1). Из равенства (4.1) и теоремы 2.5 следует, что функция $\varphi_{n}^{+}(x, \lambda)$ при $|x| \rightarrow \infty$ имеет асимптотику

$$
\begin{aligned}
\varphi_{n}^{+}(x, \lambda)=- & \frac{i}{2 t \sqrt{\lambda}} \exp (\lambda t+i|x| \sqrt{\lambda}) \\
& \times \int_{-\infty}^{+\infty} \exp (\mp i y \sqrt{\lambda})\left(g_{n} u_{n}^{+}\right)(y, \lambda) d y+o(1), \quad x \rightarrow \pm \infty
\end{aligned}
$$

поэтому определенная равенством (4.2) функция $u_{n}^{+}(x, \lambda)$ действительно является решением задачи рассеяния, причем из (4.3) следует, что коэффициенты прохождения и отражения могут быть выражены по формулам

$$
\begin{aligned}
& T_{n}^{+}(\lambda)=1-\frac{i \exp (\lambda t)}{2 t \sqrt{\lambda}} \int_{-\infty}^{+\infty} \exp (-i y \sqrt{\lambda})\left(g_{n} u_{n}^{+}\right)(y, \lambda) d y \\
& R_{n}^{+}(\lambda)=-\frac{i \exp (\lambda t)}{2 t \sqrt{\lambda}} \int_{-\infty}^{+\infty} \exp (i y \sqrt{\lambda})\left(g_{n} u_{n}^{+}\right)(y, \lambda) d y
\end{aligned}
$$

Аналогичные формулы справедливы и для решения $u_{n}^{-}(x, \lambda)$

$$
\begin{aligned}
u_{n}^{-}(x, \lambda) & =\exp (-i x \sqrt{\lambda})+\varphi_{n}^{-}(x, \lambda), \\
\varphi_{n}^{-}(\cdot, \lambda) & =F_{n}(\lambda)\left(\exp (-i(\cdot) \sqrt{\lambda})+\varphi_{n}^{-}(\cdot, \sqrt{\lambda})\right), \\
R_{n}^{-}(\lambda) & =-\frac{i \exp (\lambda t)}{2 t \sqrt{\lambda}} \int_{-\infty}^{+\infty} \exp (-i y \sqrt{\lambda})\left(g_{n} u_{n}^{-}\right)(y, \lambda) d y .
\end{aligned}
$$


Напомним (см. [1]), что из общей теории следует равенство $T_{n}^{+}(\lambda)=T_{n}^{-}(\lambda)$.

Из теоремы 2.6 и определения (4.2) следует, что равномерно по $\lambda$ на компактах в $(0, \infty) \backslash\left\{\lambda_{j}\right\}$ в метрике $L^{2}(\mathbb{R}, \exp (-a|x|) d x)$

$$
u_{n}^{ \pm}(x, \lambda) \rightarrow u_{\infty}^{ \pm}(x, \lambda), \quad n \rightarrow \infty,
$$

а воспользовавшись уравнением (2.11) и леммой 2.1, мы получим, что (4.7) справедливо в каждой точке $x$ равномерно по $\lambda$.

Лемма 4.1. Сходимость в (4.7) осуществляется в $C^{1}([a, b])$, әде $[a, b]-$ любой отрезок, принадлежсащий дополнению множества $\omega$, причем функиии $u_{\infty}^{ \pm}(x, \lambda)$ равны нулю, если $x \in \omega 2, \lambda \notin\left\{\lambda_{j}\right\}$.

ДокАЗАТЕльство. Последнее утверждение есть очевидное следствие уравнения (2.11), которому удовлетворяют функции $u_{\infty}^{ \pm}(x, \lambda)$ при $n=\infty$, а для доказательства первого утверждения достаточно взять принадлежаший дополнению множества $\omega$ отрезок $[c, d], c<a, b<d$, и заметить, что при достаточно большом $n$ функции $u_{n}^{ \pm}(x, \lambda)$ есть решение задачи

$$
\begin{gathered}
-\partial_{x x}^{2} u_{n}^{ \pm}(x, \lambda)+V_{\infty}(x) u_{n}^{ \pm}(x, \lambda)=0, \quad c<x<d, \\
u_{n}^{ \pm}(c+0, \lambda)=u_{n}^{ \pm}(c, \lambda), \\
u_{n}^{ \pm}(d-0, \lambda)=u_{n}^{ \pm}(d, \lambda),
\end{gathered}
$$

поэтому из сходимости функций $u_{n}^{ \pm}(x, \lambda)$ в точках $c, d$ следует их сходимость в метрике $C^{1}([a, b])$.

\section{§5. Поведение коэффициентов рассеяния и прохождения в окрестности резонанса}

Лемма 5.1. Равномерно по $\lambda$ на компактах в $(0, \infty) \backslash\left\{\lambda_{j}\right\}$

$$
T_{n}^{ \pm}(\lambda) \rightarrow 0, \quad\left|R_{n}^{ \pm}(\lambda)\right| \rightarrow 1, \quad n \rightarrow \infty .
$$

ДоКАЗАТЕЛЬСТво. Как известно [1], для любой точки $x$ справедливо равенство

$$
-2 i \sqrt{\lambda}\left|T_{n}^{ \pm}(\lambda)\right|^{2}=u_{n}^{ \pm}(x, \lambda) \partial_{x} \overline{u_{n}^{ \pm}(x, \lambda)}-\overline{u_{n}^{ \pm}(x, \lambda)} \partial_{x} u_{n}^{ \pm}(x, \lambda) .
$$

Возьмем в этом равенстве точку $x$ из внутренности множества $\omega 2$ и воспользуемся леммами 4.1 и 4.2. Получим первое из соотношений (5.1). Второе следует из унитарности матрицы рассеяния (см. [1]).

Лемма 5.2. Если $\varepsilon>0$ и $n_{0}<\infty$ выбраны в соответствии с теоремой 3.5 , то при $n>n_{0} u\left|\lambda-\lambda_{j}\right|<\varepsilon$ решение задачи рассеяния представимо в виде:

$$
u_{n}^{ \pm}(\cdot, \lambda)=\frac{w_{n}^{ \pm}(\lambda) \psi(\cdot, \lambda, n)}{C_{n}(\lambda)\left(1-\mu_{n}(\lambda)\right)}+u_{\mathrm{reg}}(\cdot, \lambda, n),
$$

əдe

$$
\begin{aligned}
w_{n}^{ \pm}(\lambda) & =-\left\langle\psi(\cdot, \lambda, n) \mid g_{n} \exp ( \pm i(\cdot) \sqrt{\lambda})\right\rangle, \\
u_{\mathrm{reg}}^{ \pm}(\cdot, \lambda, n) & =\exp ( \pm i(\cdot) \sqrt{\lambda})+S_{n}(\lambda) F_{n}(\lambda) \exp ( \pm i(\cdot) \sqrt{\lambda}) .
\end{aligned}
$$

Доказательство этого утверждения получается прямой выкладкой на основе формул (4.1), (4.2) и теоремы 3.5 .

Используя формулы (5.2), (4.4), (4.6), мы получаем следующую лемму. 
ЛЕмма 5.3. При выполнении условий леммы 5.2 справедливы формуль

$$
T_{n}^{ \pm}(\lambda)=-\frac{i \exp (\lambda t) w_{n}^{+}(\lambda) w_{n}^{-}(\lambda)}{2 t \sqrt{\lambda} C_{n}(\lambda)\left(1-\mu_{n}(\lambda)\right)}+T_{\mathrm{reg}}^{ \pm}(\lambda, n),
$$

2дe

$$
\begin{gathered}
T_{\mathrm{reg}}^{+}(\lambda, n)=1-\frac{i \exp (\lambda t)}{2 t \sqrt{\lambda}} \int_{-\infty}^{+\infty} \exp (i y \sqrt{\lambda})\left(g_{n} u_{\mathrm{reg}}^{+}(\cdot, \lambda, n)\right)(y) d y \\
R_{n}^{ \pm}(\lambda)=-\frac{i \exp (\lambda t) w_{n}^{ \pm}(\lambda)^{2}}{2 t \sqrt{\lambda} C_{n}(\lambda)\left(1-\mu_{n}(\lambda)\right)}+R_{\mathrm{reg}}^{ \pm}(\lambda, n)
\end{gathered}
$$

əдe

$$
R_{\mathrm{reg}}^{ \pm}(\lambda, n)=-\frac{i \exp (\lambda t)}{2 t \sqrt{\lambda}} \int_{-\infty}^{+\infty} \exp ( \pm i y \sqrt{\lambda})\left(g_{n} u_{\mathrm{reg}}^{ \pm}(\cdot, \lambda, n)\right)(y) d y .
$$

Из теоремы 3.5 и формул $(5.4),(5.5),(5.8)$ следует

Лемма 5.4. При $\lambda \notin\left\{\lambda_{j}\right\}$ существуют предель

$$
\lim _{n \rightarrow \infty} T_{n}^{ \pm}(\lambda)=0, \quad \lim _{n \rightarrow \infty} R_{n}^{ \pm}(\lambda)=R_{\infty}^{ \pm}(\lambda),
$$

и равномерно по $\lambda$ в круге $\left|\lambda-\lambda_{j}\right|<\varepsilon$ существуют предель

$$
\begin{aligned}
& \lim _{n \rightarrow \infty} T_{\text {reg }}^{ \pm}(\lambda, n)=T_{\text {reg }}^{ \pm}(\lambda, \infty), \\
& \lim _{n \rightarrow \infty} R_{\text {reg }}^{ \pm}(\lambda, n)=R_{\text {reg }}^{ \pm}(\lambda, \infty) .
\end{aligned}
$$

Лемма 5.5. Справедливы равенства

$$
\begin{aligned}
& T_{\mathrm{reg}}^{ \pm}\left(\lambda_{j}, \infty\right)=0, \\
& R_{\mathrm{reg}}^{ \pm}\left(\lambda_{j}, \infty\right)=\lim _{\left|\lambda-\lambda_{j}\right| \rightarrow 0}\left(\lim _{n \rightarrow \infty} R_{n}^{ \pm}(\lambda)\right) .
\end{aligned}
$$

ДОКАЗАТЕЛЬСТвО. При $\lambda \notin\left\{\lambda_{j}\right\}$

$$
T_{\infty}^{ \pm}(\lambda)=\lim _{n \rightarrow \infty} T_{n}^{ \pm}(\lambda)=0=-\frac{i \exp (\lambda t) w_{\infty}^{+}(\lambda) w_{\infty}^{-}(\lambda)}{2 t \sqrt{\lambda} C_{\infty}(\lambda)\left(1-\mu_{\infty}(\lambda)\right)}+T_{\text {reg }}^{ \pm}(\lambda, \infty) .
$$

В силу следствия 3.3 функция

$$
\lambda \rightarrow C_{\infty}(\lambda)\left(1-\mu_{\infty}(\lambda)\right)
$$

аналитична и в точке $\lambda_{j}$ имеет нуль первого порядка.

Функции

$$
\lambda \rightarrow w_{\infty}^{ \pm}(\lambda)
$$

аналитичны и в силу следствия 3.1 в точке $\lambda_{j}$ равны нулю. Поэтому

$$
T_{\text {reg }}^{ \pm}\left(\lambda_{j}, \infty\right)=\lim _{\left|\lambda-\lambda_{j}\right| \rightarrow 0} T_{\text {reg }}^{ \pm}(\lambda, \infty)=0 .
$$

Аналогично, при $\lambda \notin\left\{\lambda_{j}\right\}$

$$
R_{\infty}^{ \pm}(\lambda)=-\frac{i \exp (\lambda t) w_{\infty}^{ \pm}(\lambda)^{2}}{2 t \sqrt{\lambda} C_{\infty}(\lambda)\left(1-\mu_{\infty}(\lambda)\right)}+R_{\text {reg }}^{ \pm}(\lambda, \infty),
$$

и в силу изложенных выше причин

$$
\lim _{\left|\lambda-\lambda_{j}\right| \rightarrow+0} R_{\infty}^{ \pm}(\lambda)=\lim _{\left|\lambda-\lambda_{j}\right| \rightarrow+0} R_{\text {reg }}^{ \pm}(\lambda, \infty) .
$$


ЛЕмма 5.6. Справедливо равенство

$$
\lim _{n \rightarrow \infty} \frac{\left|w_{n}^{+}\left(\operatorname{Re} \lambda_{j, n}\right)\right|^{2}+\left|w_{n}^{-}\left(\operatorname{Re} \lambda_{j, n}\right)\right|^{2}}{\operatorname{Im} \lambda_{j, n}}=-4 \sqrt{\lambda_{j}} t^{2} \exp \left(-2 \lambda_{j} t\right) .
$$

ДоКАЗАТЕЛЬСТВо. Из теоремы 3.1 следует, что

$$
\begin{aligned}
& \frac{\left|w_{n}^{+}\left(\operatorname{Re} \lambda_{j, n}\right)\right|^{2}+\left|w_{n}^{-}\left(\operatorname{Re} \lambda_{j, n}\right)\right|^{2}}{\operatorname{Im} \lambda_{j, n}} \\
& =-4 t \sqrt{\operatorname{Re} \lambda_{j, n}} \exp \left(-\operatorname{Re} \lambda_{j, n} t\right) \\
& \quad \times\left\langle g_{n} \psi\left(\cdot, \operatorname{Re} \lambda_{j, n}, n\right) \mid \overline{\psi\left(\cdot, \operatorname{Re} \lambda_{j, n}, n\right)}\right\rangle \frac{\operatorname{Im} \mu_{n}\left(\operatorname{Re} \lambda_{j, n}\right)}{\operatorname{Im} \lambda_{j, n}} .
\end{aligned}
$$

Ho

$$
\begin{aligned}
\mu_{n}\left(\operatorname{Re} \lambda_{j, n}\right)-1 & =\mu_{n}\left(\operatorname{Re} \lambda_{j, n}\right)-\mu_{n}\left(\lambda_{j, n}\right) \\
& =-\frac{i \partial \mu_{n}\left(\operatorname{Re} \lambda_{j, n}\right)}{\partial \lambda} \operatorname{Im} \lambda_{j, n}+O\left(\left(\operatorname{Im} \lambda_{j, n}\right)^{2}\right),
\end{aligned}
$$

и, учитьвая лемму 3.2 , мы из (5.16) получаем формулу (5.15).

Заметим, что из (5.17) следует

Лемма 5.7. Справедливо соотношение

$$
\frac{\operatorname{Im} \lambda_{j, n} \exp \left(\operatorname{Re} \lambda_{j, n} t\right)}{2 t \sqrt{\operatorname{Re} \lambda_{j, n}} C_{n}\left(\operatorname{Re} \lambda_{j, n}\right)\left(1-\mu_{n}\left(\operatorname{Re} \lambda_{j, n}\right)\right)} \rightarrow \frac{i \exp \left(2 \lambda_{j} t\right)}{2 t^{2} \sqrt{\lambda_{j}}}, \quad n \rightarrow \infty .
$$

Положим

$$
\text { v.p. } R_{\infty}^{ \pm}\left(\lambda_{j}\right)=\lim _{\left|\lambda-\lambda_{j}\right| \rightarrow+0}\left(\lim _{n \rightarrow \infty} R_{n}^{ \pm}(\lambda)\right) \text {. }
$$

Tеорема 5.1. Справедливо равенство

$$
\lim _{n \rightarrow \infty}\left(\mid R_{n}^{+}\left(\operatorname{Re} \lambda_{j, n}\right)-\text { v.p. } R_{\infty}^{+}\left(\lambda_{j}\right)|+| R_{n}^{-}\left(\operatorname{Re} \lambda_{j, n}\right)-\text { v.p. } R_{\infty}^{-}\left(\lambda_{j}\right) \mid\right)=2 .
$$

ДокАЗАТЕЛЬСТво. Из (5.7) следует, что справедливо неравенство

$$
\begin{aligned}
& || R_{n}^{+}\left(\operatorname{Re} \lambda_{j, n}\right)-\text { v.p. } R_{\infty}^{+}\left(\lambda_{j}\right)|+| R_{n}^{-}\left(\operatorname{Re} \lambda_{j, n}\right)-\text { v.p. } R_{\infty}^{-}\left(\lambda_{j}\right) \mid-\exp \left(\operatorname{Re} \lambda_{j, n} t\right) \\
& \times\left(\left|w_{n}^{+}\left(\operatorname{Re} \lambda_{j, n}\right)\right|^{2}+\left|w_{n}^{-}\left(\operatorname{Re} \lambda_{j, n}\right)\right|^{2}\right) \frac{1}{2 t \sqrt{\operatorname{Re} \lambda_{j, n}}\left|C_{n}\left(\operatorname{Re} \lambda_{j, n}\right)\left(1-\mu_{n}\left(\operatorname{Re} \lambda_{j, n}\right)\right)\right|} \mid \\
& \leqslant \mid R_{\text {reg }}^{+}\left(\operatorname{Re} \lambda_{j, n}, n\right)-\text { v.p. } R_{\infty}^{+}\left(\lambda_{j}\right)|+| R_{\text {reg }}^{-}\left(\operatorname{Re} \lambda_{j, n}, n\right)-\text { v.p. } R_{\infty}^{-}\left(\lambda_{j}\right) \mid
\end{aligned}
$$

Функции $R_{\mathrm{reg}}^{ \pm}(\lambda, n)$ непрерьвны по $\lambda$ равномерно по $n$, поэтому, используя (5.13) и соотношения (5.15), (5.18), мы получаем утверждение теоремы.

Отметим, что формально теорема 5.1 есть следствие доказанной в [6] теоремы 1 , хотя условия, налагаемые в [6], в нашем случае не выполнены. 
ТЕОРема 5.2. Если последовательность $V_{n}$ состоит из четных функций:

$$
V_{n}(-x)=V_{n}(x),
$$

mo

$$
\lim _{n \rightarrow \infty}\left|T_{n}^{ \pm}\left(\operatorname{Re} \lambda_{j, n}\right)\right|=1 .
$$

ДоКАЗАТЕЛЬСТво. Если выполнено равенство (5.19), то

$$
\left|w_{n}^{-}(\lambda)\right|=\left|w_{n}^{+}(\lambda)\right|,
$$

поэтому

$$
\begin{array}{r}
\left|T_{n}^{ \pm}\left(\operatorname{Re} \lambda_{j, n}\right)-T_{\mathrm{reg}}^{ \pm}\left(\operatorname{Re} \lambda_{j, n}\right)\right|=\left(\left|w_{n}^{+}\left(\operatorname{Re} \lambda_{j, n}\right)\right|^{2}+\left|w_{n}^{-}\left(\operatorname{Re} \lambda_{j, n}\right)\right|^{2}\right) \\
\times 4 t \sqrt{\operatorname{Re} \lambda_{j, n}}\left|C_{n}\left(\operatorname{Re} \lambda_{j, n}\right)\left(1-\mu_{n}\left(\operatorname{Re} \lambda_{j, n}\right)\right)\right|,
\end{array}
$$

и равенство (5.20) получается из (5.21) предельнгм переходом на основе (5.15) и леммы 5.4 .

В случае несимметричного барьера соотношение (5.20) может быть и не выполнено, но можно показать, что если пределы существуют, то

$$
\begin{aligned}
\lim _{n \rightarrow \infty}\left(\mid R_{n}^{+}\left(\operatorname{Re} \lambda_{j, n}\right)-\text { v.p. } R_{\infty}^{+}\left(\lambda_{j}\right)|| R_{n}^{-}\left(\operatorname{Re} \lambda_{j, n}\right)-\text { v.p. } R_{\infty}^{-}\left(\lambda_{j}\right) \mid\right) \\
=\lim _{n \rightarrow \infty}\left|T_{n}^{ \pm}\left(\operatorname{Re} \lambda_{j, n}\right)\right|^{2} .
\end{aligned}
$$

Для оценки решения задачи рассеяния вблизи резонанса рассмотрим интеграл

Положим

$$
I(\lambda, n)=\int_{\omega 2}\left|u_{n}^{+}(x, \lambda)\right|^{2} d x+\int_{\omega 2}\left|u_{n}^{-}(x, \lambda)\right|^{2} d x .
$$

$$
\begin{aligned}
& d(n)=\frac{1}{4} \operatorname{dist}\left(\left\{x: V_{\infty}(x) \leqslant n\right\},\left\{x: V_{\infty}(x)=\infty\right\}\right), \\
& \theta(n)=\frac{\sqrt{n}}{d(n)} \exp (-d(n) \sqrt{n}) .
\end{aligned}
$$

Теорема 5.3. Справедливо неравенство

$$
I\left(\operatorname{Re} \lambda_{j, n}, n\right) \geqslant \frac{\text { const }}{\left|\operatorname{Im} \lambda_{j, n}\right|},
$$

а если последовательность $\sigma(n)$ удовлетворяет условиям:

$$
\sigma(n)>\left|\lambda_{j}-\operatorname{Re} \lambda_{j, n}\right|, \quad \sigma(n) \rightarrow 0, \quad \frac{\sigma(n)}{\theta(n)} \rightarrow \infty, \quad n \rightarrow \infty,
$$

mo

$$
\int_{\left|\lambda-\lambda_{j}\right|<\sigma(n)} I(\lambda, n) d \lambda \geqslant \text { const }>0 .
$$

ДокАЗАТЕЛЬство. Первое утверждение есть следствие неравенства Бесселя и формул (5.2) и (5.18), а второе утверждение доказано в [7] для случая размерности пространства больше двух, обобшение на рассматриваемый случай тривиально.

Заметим, что, следуя проведенным в [7] рассуждениям, можно показать, что

$$
\left|\lambda-\lambda_{j, n}\right|<\operatorname{const} \theta(n) .
$$

Более того, в рассматриваемом нами случае “толстых стенок" величина $d(n)$ в приведенных выше оценках может быть заменена на константу, однако доказательство этого факта мы отложим до другой публикации. 


\section{Список литературы}

1. Додд Р., Эйбек Дж., Гиббон Дж., Моррис Х. Солитоны и нелинейные волновые уравнения. М.: Мир, 1988.

2. Саймон Б. Модель $P(\varphi)_{2}$ евклидовой квантовой теории поля. М.: Мир, 1976.

3. Като T. Теория возмущений линейных операторов. М.: Мир, 1972.

4. Арсеньев $A$. А. О резонансных свойствах амплитуды рассеяния для резонатора Гельмгольца // Матем. моделирование. 1994. Т. 6. №6. С. 47-66.

5. Арсеньев $A$. А. О резонансных свойствах амплитуды рассеяния для уравнения Шрёдингера с ловушечным потенциалом // ТМФ. 1995. Т. 104. № 2. С. 214-232.

6. Арсеньев A.А. Резонансы матрицы рассеяния и квазиуровни // ТМФ. 1996. Т. 106. № 1. C. 16-23.

7. Арсеньев А. А. Сингулярные потенциалы и резонансы. М.: Изд-во МГУ, 1974.

8. Хилле Э., Филлипс P. Функционалњный анализ и полугруппы. М.: ИЛ, 1962.

Московский государственный

университет им. М.В. Ломоносова
Поступила в редакцию 09.12 .1994 